\title{
LA EPISTEMOLOGÍA NATURALIZADA DE LOS PRINCIPIOS JURÍDICOS
}

\section{THE NATURALIZED EPISTEMOLOGY OF LEGAL PRINCIPLES}

\author{
Jorge Luis Godenzi Alegre*
}

\section{Resumen}

Desde su radicalidad la epistemología naturalizada tiene una perspectiva notablemente diferente a la epistemología tradicional, en lo que concierne a la explicación, orientación y justificación de las disciplinas del conocimiento.

Al apelar a la filosofía analítica la epistemología naturalizada, aplicada a las ciencias jurídicas, postula que para los efectos de conocer algún concepto del derecho y de las categorías que se desprenden de esta disciplina, es necesario establecer cuáles son las creencias que se tienen sobre dichas categorías, una de ellas, la de los principios jurídicos, consiste en señalar su primacía y vigencia, de una parte, y de la otra determinar si coinciden con lo que ocurre en su aplicación y, por lo tanto, si es justificado sostener tales creencias.

Para alcanzar el estatuto epistemológico de explicación y justificación, estas creencias deben satisfacer una cantidad finita de condi-

* Investigador y Catedrático Universitario E-mail: jgodenzzi@yahoo.es ciones necesarias y suficientes. Las condiciones necesarias, estipuladas mediante los criterios que se emplean para precisar la noción que se tiene respecto de los principios jurídicos, consisten en advertir el sentido que se tiene respecto del derecho. La creencia, abrumadoramente mayoritaria, está orientada a establecer que la ley y el derecho son lo mismo; pero cabría estipular, desde una condición suficiente, reconocer que en el sustrato de esta disciplina del conocimiento, para explicarla, orientarla y justificarla mejor, es que tanto la ley como el derecho son nociones totalmente diferentes; el derecho es más relevante que la ley por que éste se nutre, entre otros aspectos, de principios que subyacen en el entramado cultural, constituyéndose como un nuevo tipo de normas que incorporan moralidad, con carácter general y abierto al ordenamiento jurídico, lo cual supone, desde ya, un frontal ataque al positivismo jurídico tradicional.

$\mathrm{Al}$ sostener esta tesis de que el derecho se encuentra por encima de las leyes, las fronteras que antaño separaban a las ciencias jurídicas de la moral se difuminan, puesto que la relevancia moral de los principios, que se pueden eventualmente invocar, constituyen en deter- 
minadas circunstancias un deber jurídico y un nuevo tipo de normas que deben ser observadas por los operadores del derecho, en las mismas condiciones que las demás normas legales. De este modo, los principios jurídicos no serán distintos de las leyes comunes y podrán ser identificados como tales, por su contenido, es decir porque moralmente son los más adecuados para justificar de modo coherente al conjunto de reglas jurídicas, y también para explicar las decisiones judiciales, cuyo norte ha de ser el de la preservación de los derechos fundamentales garantizando en un mínimo, la seguridad jurídica y la paz social.

Este enfoque naturalizado de la epistemología puede ser capaz de resolver una serie de problemas que se han adquirido con la antigua epistemología tradicional, tales como el problema de los enunciados normativos, el indeterminismo de las lagunas del derecho, la interpretación legal, las zonas de penumbra, etc.

El planteamiento y refutación de criterios epistemológicos basados en condiciones necesarias y suficientes es lo que estimula a este artículo académico que aspira a replantear nuevas bases para la explicación y justificación de los principios jurídicos.

\section{Palabras clave}

Ley - Principios - Reglas - Ponderación - Optimización - Moral - Derecho - Positivismo - Moralidad - Epistemología

\section{Abstract}

From its radicality the naturalized epistemology has a remarkably different perspective to the traditional epistemology, as far as the explanation, orientation and justification of the disciplines of the knowledge.
In appealing to analytical philosophy, naturalized epistemology, applied to the juridical sciences, postulates that for the purposes of knowing some concept of law and the categories that emanate from this discipline, it is necessary to establish what are the beliefs about them Categories, one of them, that of legal principles, is to indicate their primacy and validity, on the one hand, and on the other, to determine if they coincide with what happens in their application and, therefore, if it is justified to sustain such beliefs .

To reach the epistemological status of explanation and justification, these beliefs must satisfy a finite number of necessary and sufficient conditions. The necessary conditions, stipulated by the criteria used to define the notion of legal principles, are to note the meaning of the law. The belief, overwhelmingly majority, is oriented to establish that the law and the law are the same; But it could be stipulated, from a sufficient condition, to recognize that in the substrate of this discipline of knowledge, to explain, guide and justify it better, is that both law and law are totally different notions; The law is more relevant than the law because it is nourished, among other aspects, of principles that underlie the cultural fabric, constituting itself as a new type of rules that incorporate morality, with a general character and open to the legal order, , From now on, a frontal attack on traditional legal positivism.

In holding this view that law is above laws, the frontiers that once separated the legal sciences from morality are blurred, since the moral relevance of the principles, which may be invoked, constitute in certain circumstances A legal duty and a new type of rules that must be observed by the operators of the right, under the same conditions as the other legal norms. Thus, the legal principles will not be different from the common laws and can be identified as such, by their content, that is to say because 
they are morally the most adequate to justify in a coherent way the set of legal rules, and also to explain the decisions Judicial, whose north must be that of the preservation of the fundamental rights guaranteeing in a minimum, legal security and social peace.

This naturalized approach to epistemology may be able to solve a number of problems that have been acquired with the old traditional epistemology, such as the problem of normative statements, indeterminism of legal gaps, legal interpretation, penumbra, etc.

The approach and refutation of epistemological criteria based on necessary and sufficient conditions is what stimulates this academic article that aspires to rethink new bases for the explanation and justification of the legal principles.

\section{Keyword}

Law - Principles - Rules - Weighting - Optimization - Moral - Law - Positivism - Morality - Epistemology

\section{Introducción}

La tan discutida y poco definida categoría de los principios jurídicos es uno de los asuntos más difíciles de resolver en la disciplina de las ciencias jurídicas. Posiblemente una de las razones consista que solo ha cobrado relevancia el significado gramatical que le han otorgado al término, puesto que si ello fuese así entonces técnicamente no debería llamarse principio, sino fundamento en razón de que un principio solo existe en las ciencias exactas (Lógica, Matemática) mientras que en las ciencias sociales, como es la disciplina del Derecho, solo existen fundamentos, y aquí es donde precisamente aparece el meollo de esta cuestión a debatir, desde la perspectiva de la epistemología natu- ralizada que planteo, y lo hacemos formulando las siguientes preguntas, que están fuertemente asidas al contexto cultural: ¿Para qué sirven los principios jurídicos? ¿Acaso no es cierto que cuando se proyectan en algún ámbito específico fracasan y terminan en nada? ¿Es que sería conveniente replantear el sentido de los principios jurídicos al momento de ponderarlos con las reglas o directrices?

En el ámbito del derecho y uno de sus más eficaces propugnadores, el alemán Robert Alexy, entre otros jusfilósofos, han venido reclamando y se han esforzado en este propósito en dotar a los principios jurídicos de prestigio y crédito que los hagan indiscutibles.

Qué duda cabe, en el derecho son esenciales y necesarios, sin ellos el ordenamiento jurídico se pierde, se debilita, no habría una estructura donde asirse, ni podrían encontrarse explicaciones o justificaciones. Constantemente se le reivindican desde muchos puntos de vista. Pero, ¿concluyen en algo?, ¿terminan en algo? $\mathrm{O}$ en qué sentido pueden los principios jurídicos llevarnos a decisiones concretas, esclareciendo las "zonas de penumbra" en la que eventualmente una ley puede encontrarse.

Esto es, dilucidar si es posible que los principios jurídicos conduzcan a una conclusión, aportando soluciones que no sean exclusivamente lugares comunes que se utilizan por conveniencia política o para favorecer intereses y objetivos contradictorios, y que nos puedan permitir encaminarnos a buen puerto, por la dificultad y ambigüedad que de suyo suscita la comprensión y alcances de esta categoría que integra la disciplina del derecho.

Sépase, en primer lugar, que los principios jurídicos se pueden dilucidar en diferentes sentidos: como elementos interpretativos, como presupuestos del derecho, como explicación del ordenamiento jurídico, como verda- 
des supremas - por ser de máxima generalidad y aceptación -, como medio auxiliar con el fin de solucionar las deficiencias de la ley (lagunas). Igualmente, resulta incuestionable que los fundamentos ontológicos de los principios jurídicos se encuentran en la naturaleza humana, racional, social y libre por lo que los tendremos en cuenta como "normas" o directrices derivadas directamente de los factores culturales por los cuales las sociedades se encuentran revestidas.

Llamo directriz a la clase de norma que establece una meta que ha de alcanzarse, generalmente en orden al perfeccionamiento de algún aspecto económico, político o social de la colectividad (si bien algunos objetivos son negativos, pues estipulan que hay que proteger de alteraciones adversas ciertos aspectos actuales). Denomino "principio" a una norma que es menester observar, no porque haga posible o asegure una situación económica, política o social que se juzgue conveniente, sino por ser un imperativo de justicia, de honestidad o de alguna otra dimensión de la moral. (Ronald Dworkin, 1980, p.85-86).

Entonces, por ser un resultado del aspecto cultural, en sus enunciados prescriptivos, existe en ellos un influyente contenido moral (mores) por lo que este elemento inexorablemente se incorporará en el ámbito jurídico. En efecto, en muchos de los textos constitucionales del siglo precedente aparecieron una serie de preceptos que aspiraban a introducir determinados postulados morales en su normatividad; $y$ así fue que se consagraron en nuevas "normas", que en no pocos casos en su estado germinal, fueron principios morales que al final quedaron juridificadas como "valores superiores". Esta experiencia definitivamente nos revela la estrecha ligazón existente entre los principios jurídicos con la moral, que muchos lo entienden como aspectos de la moralidad social. Es el caso de algunos de los principios jurídicos que en sentido estricto se han caracterizado precisamente por contener valores "superiores" en el ordenamiento jurídico. Estos valores son, a decir: "las opciones ético-sociales básicas que presiden un orden político, jurídico, económico y cultural", con una triple dimensión: fundamentadora, orientadora y crítica del ordenamiento jurídico. (Antonio Pérez Luño, 1984 pp.288-289). Desde este punto de vista, estos valores superiores se encuentran formalizados a través de los denominados derechos fundamentales, habiéndose constituido el ordenamiento jurídico como "un orden simbólico en el que se expresan la identidad y forma de vida de una comunidad jurídica particular (Jürgen Habermas, 1998, p.329).

La peculiaridad y originalidad de esta urdimbre conceptual lógicamente ha residido en la positivización de esos valores dentro del texto constitucional como una categoría nítidamente diferenciada. Es decir, aquellos valores superiores han ido adquiriendo y ostentando su carácter normativo (entiéndase que no son consejos u orientaciones sino que son directrices obligatorias para todos los ciudadanos) desde el momento en que se incorporan en la normativa vigente. Esos valores superiores representan los ideales que una sociedad ha decidido erigir como sus máximos objetivos a desarrollarse en el plano jurídico; por tanto son expresión de la moralidad mayoritariamente aceptada en el entorno cultural que al tener esa procedencia, no se agotan simplemente en su contenido normativo, sino que tales valores cumplen también una función crítica y de presión social. Su fundamento, como decíamos en líneas precedentes, es racional e histórico porque proviene del consenso social básico que tiende a realizar los objetivos fundamentales del Estado. Es menester citar, por ejemplo, que todas esas especificaciones encuentran, como punto central, en los razonamientos que despliega el actual Tribunal Constitucional que los custodia y los pondera en su aplicación al 
momento de expedir sus sentencias. Estos valores superiores, contenidos en determinados principios jurídicos, son en buena medida la expresión de una auténtica legitimidad del sistema político y jurídico en razón que de ellos se extraen las razones éticas para que todos los ciudadanos, sin excepción, permanezcan subordinados al imperio de la ley.

\section{Los límites y el desarrollo de los principios} jurídicos con las reglas

A la vista de lo anterior, detengámonos en el estudio de los principios jurídicos, como un aspecto de la teoría del derecho, relacionándolos con las "normas", capaces de proporcionar respuestas ante casos conflictivos, que por distintas circunstancias no encuentran solución en las reglas del derecho o en las normas jurídicas, constituyéndose, en muchas ocasiones, en eficaces instrumentos puesto a disposición, en cuanto adscritos y deudores de un sistema jurídico del cual nuestro ordenamiento se entronca. Podemos afirmar que en la experiencia, los principios jurídicos desempeñan un papel, como medios o presupuestos del conocimiento del derecho (ratio cognoscendi), como aspectos fundamentales del contenido del derecho (ratio essendi), como reglas de la específica técnica de interpretación y de aplicación del derecho (ratios procedendi).

Este aspecto de la teoría del derecho aparenta simpleza, sin embargo exterioriza cierta complejidad como vamos a ver a continuación y es lo que la doctrina acuña como una amplia gama de conceptos, significados y clases de principios jurídicos que podría ser, y de hecho son, ampliamente debatidos.

Podríamos distinguir siete unidades de significación en el lenguaje ordinario del término "principio": 1) parte importante de algo, propiedad fundamental, núcleo básico o ca- racterística central; 2) regla, guía, orientación o indicación general; 3) fuente generadora, causa u origen; 4) finalidad, objetivo, propósito o meta; 5) premisa o punto de partida de un razonamiento, axioma o verdad teórica evidente, esencia, propiedad definitoria; 6) regla práctica de contenido evidente, o verdad ética incuestionable; 7) máxima, aforismo, proverbio, brocardo, etc. En el ámbito jurídico, estas unidades de significación han dado lugar al uso del término "principio" en contextos y con sentidos bien diferentes (hasta once enumera este autor), en relación a las diversas actividades jurídicas, ya sean de carácter expositivo, crítico, justificativo, o de reforma e interpretación del propio Derecho. (Genaro Carrió, 1970, p. 32 y ss.). Son estándares que presentan dos características básicas: su estructura abierta y su discutible juridicidad. (García Figueroa, 1998 pp. 41 y ss.). Así, suele afirmarse que se trata de disposiciones de carácter general y fundamental, pero lo cierto es que ambas notas sólo se desprende de un posible juicio de comparación con otros tipos de enunciados jurídicos menos generales o fundamentales, respecto a los cuales ejercen o actúan como principios. En este sentido, los principios son un concepto racional o comparativo, ya que tanto "la generalidad como la fundamentalidad, que son dos rasgos comúnmente atribuidos a los principios, representan un buen ejemplo: ambas son nociones graduales y no existe ninguna regla o convención que indique cuánta generalidad o fundamentalidad se precisa para dar vida a un principio; sencillamente, se tiende a decir que una norma es un principio cuando, comparándola con otra, resulta más general o fundamental. Por este motivo, su definición sólo puede ser estipulativa o convencional. (Luis Prieto S. 2005 pp.24-25).

La noción de principio jurídico, por lo tanto, puede ser usada en diversos sentidos, entre los que se encuentran los siguientes: a) norma muy general, que regula un caso cuyas 
propiedades relevantes son muy generales; b) norma redactada en términos particularmente vagos; c) norma que expresa los valores superiores de un ordenamiento jurídico, de un sector del mismo o de una institución; d) norma programática o directriz, esto es, que estipula la obligación de perseguir determinados fines; e) norma dirigida a los órganos de aplicación del Derecho, que señala con carácter general cómo se debe seleccionar la norma aplicable y cómo interpretarla; y f) enunciado o máxima (regula iuris) de un considerable grado de generalidad, que permite la sistematización del ordenamiento o de un sector del mismo. (Ruiz Manero, 1996 pp.150-151).

La convivencia entre los principios jurídicos, las reglas y las directrices

Conviene señalar que algunos de los estudios más recientes sobre los principios jurídicos tienden a centrarse e incidir en el tema de la distinción estructural y funcional con las reglas. Al respecto, pueden encontrarse tres tesis básicas sobre esta diferenciación: una primera que niega tal separación, debido a la pluralidad jurídica realmente existente; una segunda postura que sólo atribuye a dicha distinción una diferencia de grado, donde la generalidad y fundamentalidad serían los criterios decisivos; $\mathrm{y}$ en tercer lugar, otra posición que permite hablar de una diferencia cualitativa y no sólo gradual entre reglas y principios, es decir, que existe un criterio que permite distinguir con toda precisión entre principios y reglas. (Robert Alexis pp.85-86).

Cuando afirmamos que los principios jurídicos son "normas" o directrices, es decir, enunciados prescriptivos que se refieren a lo que debe ser, tal como sugiere Robert Alexy, pueden ser formulados mediante los operadores deónticos del mandato, de la prohibición, o de la permisión. (Robert Alexis, 1993 pp.83).
Este mismo autor entiende los principios jurídicos como mandatos de optimización de la siguiente manera, conviene textualmente transcribirlo:

El punto decisivo para la distinción entre reglas y principios es que los principios son normas que ordenan que se realice algo en la mayor medida posible, en relación con las posibilidades jurídicas y fácticas. Los principios son, por consiguiente, mandatos de optimización que se caracterizan porque pueden ser cumplidos en diversos grados y porque la medida ordenada de su cumplimiento no sólo depende de las posibilidades fácticas, sino también de las posibilidades jurídicas. El campo de las posibilidades jurídicas está determinado a través de principios y reglas que juegan en sentido contrario.....En cambio, las reglas son normas que exigen un cumplimiento pleno y, en esa medida, pueden siempre ser sólo o cumplidas o incumplidas. Si una regla es válida, entonces es obligatoria hacer precisamente lo que ordena, ni más ni menos. Las reglas contienen por ello determinaciones en el campo de lo posible fáctica y jurídicamente. Lo importante por ello no es si la manera de actuar a la que se refiere la regla puede o no ser realizada en distintos grados. Hay por tanto distintos grados de cumplimiento. Si se exige la mayor medida posible de cumplimiento en relación con las posibilidades jurídicas y fácticas, se trata de un principio. Si sólo se exige una determinada medida de cumplimiento, se trata de una regla. (Robert Alexy, Robert, 1993, pp.86-87).

Sobre estas diferencias, también afloran las opiniones de otros autores, que han sostenido la distinción entre reglas y principios en función de la distinta formas que se puedan utilizar para resolver conflictos o cuando brotan ásperas contradicciones entre dos reglas o dos principios. Así, el propio Ronald Dworkin ha señalado que los principios poseen una dimensión de peso o de importancia de la que carecen las reglas. Una contradicción entre 
principios ha de resolverse dando preferencia a uno de ellos, siendo ambos válidos, mientras que una antinomia entre reglas se soluciona aplicando sólo una de ambas, dado que la otra resulta inválida. (Ronald Dworkin, 2012, p.78).

En un sentido similar, y al menos como punto de partida, Robert Alexy distingue entre conflictos de reglas y colisiones de principios, según el modo de solucionar la situación de incompatibilidad normativa en uno u otro caso. En este sentido, un conflicto de reglas se resuelve o bien introduciendo en una de las reglas una cláusula de excepción que elimina el conflicto, o declarando inválida una de ellas.

Una norma vale o no vale jurídicamente. Que una regla vale y es aplicable a un caso significa que vale también su consecuencia jurídica. Cualquiera que sea la forma cómo se los fundamente, no puede ser el caso que valgan dos juicios concretos de deber ser recíprocamente contradictorios. Si se constata la aplicabilidad de dos reglas con consecuencia recíprocamente contradictorias en el caso concreto y esta contradicción no puede ser eliminada mediante la introducción de una cláusula de excepción, hay entonces que declarar inválida por lo menos una de las reglas. (Robert Alexy, 1993, p. 88).

En sentido opuesto, las colisiones entre principios deben resolverse con la cesión de uno ante el otro, pero esto no significa que haya que introducir una cláusula de excepción, ni que el principio no aplicado deba ser declarado inválido, sino que bajo ciertas circunstancias, uno de los principios se aplica con preferencia al otro. De esta manera, el conflicto se resuelve mediante la ponderación entre los principios en contradicción, teniendo en cuenta las circunstancias del caso para establecer una relación de preferencia condicionada. Se trata, por lo tanto, de la ponderación entre intereses opuestos en un caso determinado y bajo ciertas condiciones de aplicación en las cuales un principio precede al otro; pero ante otras condiciones diferentes, la preferencia puede darse de forma inversa, en función del peso específico que tenga cada uno de los principios. Frente a la noción de "contradicción" aplicable a las reglas, otros autores prefieren hablar de "contraposiciones" entre principios, puesto que su característica sería precisamente dar lugar a soluciones contrapuestas cuando cada principio se toma por separado. (Claus Canaris, 199, pp.130-131).

Todo ello trae como consecuencia que las reglas y los principios sean razones que deciden de un modo diferente: los principios son siempre razones prima facie, en cambio las reglas, a menos que se haya establecido una excepción, son razones definitivas (Jaap Hage, 1997 pp.115-118). En este aspecto se apartaría de Alexy de Dworkin; el primero pondría en duda que las reglas aparezcan siempre bajo la pauta de "todo o nada", dado que sería posible ir incluyendo nuevas excepciones en los supuestos o condiciones de aplicación de las normas, con la aparición de nuevos casos o hechos. Además, la dimensión de peso de los principios, como ha apuntado Hage en respuesta crítica a Dworkin, surge cuando ingresa en conflicto con otras normas, pero no antes, ya que siempre hay "razones" en juego no decisivas; en cambio, las reglas sí que contienen un número finito de razones para decidir, de forma excluyente.

Esto es en las reglas se excluye que al producirse un hecho condicionante, no tenga lugar la consecuencia jurídica prevista. Precisamente a ello se refiere Alexy cuando manifiesta:

Los principios ordenan que algo deba ser realizado en la mayor medida posible, teniendo en cuenta las posibilidades jurídicas y fácticas. Por lo tanto, no contienen mandatos definitivos sino sólo prima facie. Del hecho de que un prin- 
cipio valga para un caso no se infiere que lo que el principio exige para este caso valga como resultado definitivo. Los principios presentan razones que pueden ser desplazadas por otras razones opuestas. El principio no determina cómo ha de resolverse la relación entre una razón y su opuesta. Por ello, los principios carecen de contenido de determinación con respecto a los principios contrapuestos y las posibilidades fácticas (....) Totalmente distinto es el caso de las reglas. Como las reglas exigen que se haga exactamente lo que en ellas se ordena. Contienen una determinación en el ámbito de las posibilidades jurídicas y fácticas, lo que puede conducir a su invalidez; pero, si tal no es el caso, vale entonces definitivamente lo que la regla dice." (Robert Alexy, 1993 p.99).

Por el contrario, los principios son siempre "condicionales derrotables" que admiten excepciones implícitas derivables de otros principios, excepciones que no pueden ser exhaustivas, y así no se excluye que incluso en presencia del hecho condicionante, la consecuencia jurídica prevista no tenga lugar, si tal consecuencia resulta ser "superada" por otro principio con el que ha entrado en colisión. (Riccardo Guastini, 2009 pp.150-151).

Para Robert Alexy, por lo tanto, lo que hace que una norma sea una regla o un principio depende del diferente modo de solucionar una contradicción normativa: si una norma cede siempre ante otra, se trata de un conflicto entre reglas, mientras que si tal cesión se produce según las circunstancias o condiciones del caso, se produce una colisión entre principios. Pero la cuestión resulta algo más compleja, puesto que algunas normas de carácter principal pueden funcionar como reglas, y algunas reglas pueden actuar como principios, según las circunstancias. Así, por ejemplo, sucede que un derecho fundamental opera como regla cuando no entra en contradicción con otro derecho fundamental, pero en el supuesto en que sí lo haga, actúa como principio frente al otro derecho fundamental. Además, este mismo autor sostiene que a partir de toda ponderación iusfundamental correcta, puede formularse una norma de derecho fundamental adscrita con el carácter de regla, bajo la cual puede ser subsumido un caso, es decir, que la determinación de una relación de preferencia termina, de acuerdo con la ley de colisión que rige entre principios contradictorios, en el establecimiento de una regla. (Robert Alexy, 1993 pp.98-103). A su vez, un principio se transformaría en regla cuando su hipotética colisión hubiera de solucionarse con la pérdida de validez de uno de los principios en contradicción, o si paradójicamente fuese reconocido como absoluto, es decir, que siempre se aplicará frente a otro principio. El primer caso se daría siguiendo el ejemplo propuesto por el propio Alexy, si en el ordenamiento jurídico alemán (o en el peruano, como también podría suceder), ingresara el principio de discriminación racial. Por otro lado, la segunda situación, es decir la defensa de principios "absolutos" tan fuertes como para desplazar a cualquier otro principio, es fácilmente criticable y rechazable en un ordenamiento jurídico que reconoce derechos fundamentales.

El primer caso se daría siguiendo el ejemplo propuesto por el propio Alexy, si en el ordenamiento jurídico alemán (o en el peruano, como también podría suceder), ingresara el principio de discriminación racial. Por otro lado, la segunda situación, es decir la defensa de principios "absolutos" tan fuertes como para desplazar a cualquier otro principio, es fácilmente criticable y rechazable en un ordenamiento jurídico que reconoce derechos fundamentales. (Robert Alexy, 1983 pp.105 y ss.).

Las posibles situaciones que, a juicio de Luis Prieto Sanchís, otro apreciado doctrinario, podrían tener lugar en esta relación dinámica y fluctuante entre reglas y principio, serían las 
siguientes: $1^{\circ}$ ) Normas que habitualmente llamamos principios, pero que pueden funcionar como reglas; $2^{\circ}$ ) Normas que los juristas suelen llamar reglas, pero que pueden funcionar como principios. A estos dos supuestos cabría añadir otras dos situaciones más bien paradójicas: $3^{\circ}$ ) un principio se convertiría en regla si fuese reconocido como absoluto; y $4^{\circ}$ ) un principio dejaría de funcionar como tal si se prevé con carácter general y estricto su orden de aplicación en caso de conflicto con otra norma. (Luis Prieto, 2002 p.59).

En otro sentido y de acuerdo con la distinción trazada por Manuel Atienza y J. Ruiz Manero entre principios en sentido estricto y principios como normas programáticas o directrices (Atienza M., Ruiz Manero, 199, pp.153 y ss.). Hay que tener en cuenta que las normas jurídicas pueden concebirse estructuralmente a partir de correlaciones entre casos genéricos o antecedentes y soluciones o consecuentes. Se trata de analizar si los principios en sentido estricto y las directrices tienen o no una estructura condicionada (es decir, que pueda correlacionar casos con soluciones), y si, por lo tanto, les son de aplicación los mismos operadores deónticos que a las reglas, para así poder descubrir posibles antinomias normativas en las que se encontrarán presentes principios jurídicos.

De esta manera, la distinción estructural entre reglas, principios en sentido estricto $y$ directrices en sentido general, se refiere a que las reglas configuran de forma cerrada tanto el supuesto de hecho como la conducta calificada deónticamente en la solución; los principios en sentido estricto configuran de forma abierta su supuesto de hecho y de forma cerrada la conducta calificada deónticamente; mientras que las directrices o normas programáticas configurarían de forma abierta tanto el supuesto de hecho como la conducta calificada deónticamente.
Teniendo en cuenta los ejemplos propuestos por los autores citados, el principio de igualdad, reconocido en el artículo 2 inciso 2 de nuestra Constitución Política, sería un principio en sentido estricto, en razón de que no admitiría modalidades graduales de cumplimiento. Este artículo establece lo siguiente: "Toda persona tiene derecho: A la igualdad ante la ley. Nadie debe ser discriminado por motivo de origen, raza, sexo, idioma, religión, opinión, condición económica o de cualquiera otra índole". Dicho enunciado, en forma condicional diría lo siguiente: "Si (condición de aplicación) un órgano jurisdiccional utilizara sus prerrogativas normativas (esto es, dicta una norma a fin de regular un caso genérico o la aplica para resolver un caso individual) y en relación con el caso individual o genérico de que se trate no concurre otro principio que, en relación con el mismo, tenga un mayor peso, entonces (solución normativa) a ese órgano le está prohibido discriminar en razones de nacimiento, raza, sexo, opinión o religión, condición económica”. Así pues, quedarían configuradas de forma abierta las condiciones de aplicación, pero de forma cerrada la descripción de la conducta prohibida: discriminar.

Esto no sucede con las directrices o normas programáticas, como es, por ejemplo, con el artículo 65 del mismo texto constitucional que establece: "El Estado defiende el interés de los consumidores y usuarios. Para tal efecto garantiza el derecho a la información sobre los bienes y servicios que se encuentran a su disposición en el mercado. Asimismo vela, en particular, por la salud y la seguridad de la población". En este enunciado se configura de forma abierta tanto las condiciones de aplicación como el modelo de conducta prescrito, puesto que no se ordena ni se prohíbe ninguna acción, sino que sólo se señala la consecución de un objetivo. Las acciones para lograr ese objetivo no se encuentran constitucionalmente determinadas. 
Por lo tanto, una norma está cerrada cuando su determinación fáctica o los posibles supuestos de hecho son exhaustivos, esto es, forman un conjunto finito y cerrado de circunstancias; por el contrario, una norma está abierta cuando carece de un catálogo tasado de condiciones de aplicación o carece de calificación deóntica. Este punto de vista estructural, no obstante, plantea algunos problemas en su formulación.

Así, se advierte que sería posible alterar la estructura (de regla o principio) de las normas sin cambios relevantes aparentes en su significado. Afirma que "es posible ordenar una misma acción bajo diversas formas, bien de regla, bien de principio, dependiendo de la distribución de la apertura. (Alfonso García Figueroa, 1998 p.151).

No parece que pueda negársele cierto carácter discrecional a los principios, en razón de que una misma disposición normativa en determinados casos puede ser interpretada como regla o como principio.

En relación al ejemplo ante citado del inciso 2 del artículo 2 de nuestra Constitución Política, el principio de igualdad está recogido de forma similar en la Constitución italiana, que establece: "Todos los ciudadanos (......) son iguales ante la ley, sin distinción de raza, sexo, lengua, religión, opiniones políticas, condiciones personales y sociales". Para Guastini, este artículo admite dos interpretaciones: como reglas y como principios. Como regla, se plasmaría de la forma siguiente: "si una ley distingue entre ciudadanos según el sexo, la raza, etc. (supuesto de hecho), entonces es constitucionalmente ilegitima (consecuencia jurídica); tal regla no admitía excepciones, y tendría un supuesto de hecho cerrado. Como principio, en cambio, la disposición anuncia de forma genérica el principio de igualdad de esta guisa: Los casos iguales deben ser tratados de un modo igual, y los casos diferentes deben ser tratados de un modo diferente"; el principio admitiría excepciones, y tendría el supuesto de hecho abierto. La opción entre ambas interpretaciones forma parte del poder discrecional del órgano decisor. (Riccardo Guastini, 1996, p.40).

En cualquier caso, en los principios siempre existe algún elemento estructural que permanece abierto hasta que es "cerrado" por el intérprete al aplicar la norma al caso concreto.

El margen de indeterminación de los principios jurídicos

Dadas estas diferencias, los principios en sentido estricto bien podrían dar lugar a contradicciones respecto a reglas y a otros principios en sentido estricto, lo que constituiría una antinomia normativa en los términos expuestos, ya que ambos tipos de disposiciones exigen su pleno cumplimiento en cuanto a la conducta calificada deónticamente; es decir, que los principios en sentido estricto presentan un grado de determinación semejante al de las reglas cuando son aplicados. Por ese motivo, desde el punto de vista de la actividad interpretativa, los procedimientos argumentativos para eliminar la vaguedad o "zona de penumbra” - en terminología hartiana - de una regla cerrada no son sustancialmente diferentes de los que se requieren para "cerrar" un principio abierto. (Luis Prieto S. 2002 p.360).

En sentido contrario, con respecto a las directrices en las que sí caben modalidades graduables de cumplimiento, puesto que configuran de forma abierta tanto su supuesto de hecho como la conducta calificada deónticamente, una posible colisión con otros enunciados normativos sólo se produciría al nivel de una contradicción axiológica o valorativa, por la propia indeterminación de la consecuencia 
jurídica, en la que incluso habría que considerar la concurrencia de otros fines y medios disponibles para la realización de los objetivos marcados en la directriz, lo que remitiría también a un problema de técnica legislativa o de delimitación de fines prioritarios por parte del legislador, y no sólo a la decisión judicial que pretendiera solucionar el conflicto. Hay que volver a tener en cuenta que los principios, en sentido estricto son normas abiertas que expresan derechos, y en caso de pretensiones en conflicto dependen en última instancia de una decisión judicial. En cambio, las directrices contienen intereses que no informan directamente de la acción o comportamiento debido, no imponen una obligación genuina, sino que forman parte de un programa político amplio, que requiere de un desarrollo legislativo y reglamentario, sin renunciar por ello al control jurisdiccional posterior. (Luis Prieto S. 1992 pp. 55-56)

En todo caso, una posible contradicción entre principios y reglas resulta más bien aparente en virtud del mayor peso de los principios, o como dicen Atienza y Ruiz Manero: La aplicabilidad de toda regla está condicionada a que su aplicación no entre en conflicto con un principio que, en relación con las propiedades relevantes del caso, tenga un mayor peso. (Atienza M., Ruiz Manero 1999 p. 33).

No obstante, hay que tener en cuenta, por sus importantes repercusiones, que cuando se encuentran en conflicto derechos fundamentales, se produce una situación excepcional e incluso paradójica: la aplicación de una regla puede ser preferente a la aplicación de un principio. La razón es muy simple: la posición preeminente en el ordenamiento jurídico que ocupan los derechos fundamentales, cuya estructura es la de principios constitucionales, hace que prevalezcan frente a principios opuestos. (Joaquín Rodríguez Toubes, 2000, pp. 35 y ss. y 121 y ss.).
No cabe duda que los principios son tendencialmente contradictorios y cuando se plantean una colisión existe un amplio margen de interpretación por parte del juez.

La importancia de la ponderación en el Derecho actual se explica a partir del "amplísimo contenido material o sustantivo de principios y derechos fundamentales tendencionalmente contradictorios, donde el modelo tradicional de resolver las colisiones entre reglas resulta inservible. La conservación íntegra de la Constitución exige ponderar porque sólo así es posible conservar en pie de igualdad abstracta normas o derechos que reflejan valores heterogéneos propios de una sociedad plural que, sin embargo, se quiere unida y consensuada en torno a la Constitución (...) Pero la técnica de la ponderación presenta una consecuencia sobresaliente, y es que fortalece la posición del juez, de todo juez y no sólo del constitucional: allí donde aparece un conflicto entre principios surge una apelación a la justificación racional de una decisión que, sólo en el caso concreto, otorga preferencia a uno $\mathrm{u}$ otro principio; justificación que puede conducir a cualquier resultado con el único límite precisamente de la irracionalidad. (Luis Prieto Sanchís, 2002 pp.47 y ss.).

Un caso ilustrativo que podemos emplear en esta ocasión es el de la periodista Magaly Medina. En este affaire se encuentra delimitada la colisión entre el derecho al honor y a la libertad de expresión. Al respecto consideramos, en primer lugar, la inexistencia de alguna relación de preferencia entre el ámbito de la libertad de expresión y el tipo penal protector del honor; en segundo lugar, ninguno de esos derechos tiene carácter absoluto.

En estos casos se impone la necesaria ponderación de ambos principios con el único límite que la decisión final del juez no sea claramente irrazonable. Sobre este y otros aspectos 
conceptuales, la reciente elaboración doctrinal ofrece un sinnúmero de posturas que merecen ser examinadas desde diversos aspectos diferenciables, pero ese despliegue analítico será materia de otro artículo académico.

Como habrá advertido paciente lector y tal como se ha podido pormenorizar, los principios jurídicos tienen cierta complejidad por su ambigüedad y versatilidad. Los estudiosos del derecho, de visión luminosa y los doctrinarios perspicaces le han dedicado una especial atención, por lo que no cabe duda en reconocerle su creciente relevancia que cumplen tanto en el ámbito teórico como en el pragmático, a tenor de la superposición de los sistemas jurídicos actuales y ante los continuos cambios tecnológicos, económicos y culturales que en el mundo y en el país en particular se vienen produciendo.

De manera que el derecho, al admitir el rol prioritario que desempeñan los principios, la moralidad legalizada se encuentra incorporada en el ordenamiento jurídico puesto que eventualmente, o se erigen en fundamentos de sentencias; o como orientadores respecto de la existencia de normas dudosas; o que se empleen como moldes hermenéuticos y todo ello constituye un aporte que habrá que estudiar, analizar, reflexionar y discutir.

Por último, el derecho debe asumir un compromiso por la justicia y con la aplicación de los principios jurídicos crear las condiciones básicas para garantizar la seguridad jurídica y por ende la ansiada paz social

\section{Conclusiones}

PRIMERA.- En la política contemporánea, en la que las ideologías se han licuado en beneficio de sensibilidades de opinión pública, el derecho se presenta no sólo como exigencia social en la solución de los conflictos de intereses sino también muestra que en épocas en las que se desbordan los límites de la sensatez, el derecho, que es realidad dinámica que incluye normas, procedimientos, valores, acciones y principios deberá siempre estar conectado con los perfiles de la sociedad. Esta jerarquía presenta una doble función: psicológica, que consiste en orientar la conducta de las personas para favorecer su adhesión espontánea al ordenamiento jurídico, creando en ellas un hábito general de obediencia; y judicial, en la medida en que sirve de directriz al juez en su tarea de aplicar valores que la sociedad ha establecido como esenciales.

SEGUNDA.- Para los efectos de considerar un principio como jurídico no es preciso que provenga de una fuente determinada sino debe contar con la aprobación social general y con la vigencia efectiva en los tribunales. Por eso tienen una enorme funcionalidad práctica porque ante ciertos supuestos, sirven para resolver conflictos con la aplicación del razonamiento lógico deductivo y además porque pueden colmar las lagunas del derecho.

TERCERA.- Los principios conservan su función ideológica y fundamentadora del sistema jurídico y tienen un carácter histórico, como experiencia jurídica, al estar objetivados en la Constitución Política que es portadora de un sistema de valores que proporciona un contenido material al sistema. Por ello, los principios y las reglas presentan dos características básicas: su estructura abierta y su juridicidad.

CUARTA.- Los principios jurídicos tienen su origen en el derecho natural y están constituidos por creencias, valores, convicciones $\mathrm{y}$ aspiraciones que se han ido formando a lo largo del tiempo hasta convertirse en tradición nacional. Todo ello se refleja en la dirección de la organización política de la comunidad por lo que su función principal es vertebradora del 
ordenamiento jurídico por su carácter básico y fundamental, al reflejar las creencias y convicciones del grupo social. Consecuentemente, un principio jurídico no es un texto frío que se proyecta en una "norma" sino que expresa un valor jurídico de la comunidad.

QUINTA.- La importancia de los principios y de las reglas estriba en que son instrumentos adecuados que sirven para comprender la estructura y funcionamiento de los valores superiores de un sistema jurídico, al tiempo de marcar las disimilitudes entre principios y reglas, que es un tópico que ocupa prioritariamente a la doctrina en tratar de esclarecer; si los principios son un tipo específico de normas jurídicas o pertenecen a un ámbito distinto al derecho, como la moral o la política. De todo ello, también surge una diferencia ontológica con las reglas. Sin embargo, por la naturaleza de sus enunciados ambos son normas jurídicas, aun cuando sean de distinta clase. Desde este punto de vista la distinción gravita en la configuración abierta -los principios- o cerrada - las reglas - de los supuestos de hecho.

SEXTA.- Los principios y las reglas cumplen con la finalidad de mantener al derecho sin fisuras ni vacíos, y en muchas ocasiones se desempeñan como normas implícitas, esto es, como si hubiese un derecho latente en el seno del propio ordenamiento jurídico. Estas normas pueden ser extraídas de otras normas explicitas a través de un procedimiento analógico.

SETIMA.- Se destaca la relevancia de los principios jurídicos por iluminar determinados aspectos del sistema a los que no se ha prestado la atención merecida. Es en esta función donde los principios manifiestan especialmente una dimensión axiológica que, en la práctica, orienta tanto el conocimiento como la interpretación y la aplicación de las normas del sistema jurídico.

OCTAVA.- La diferencia entre principios y reglas es comúnmente resuelta de la siguiente manera: Las reglas estarían expresadas en forma más precisa y determinada que los principios, caracterizados por la amplitud de los supuestos regulados, es decir, por una mayor eficacia cuantitativa que las reglas. Al ser más generales se aplican a más casos. En la aplicación de los principios no hay subsunción sino ponderación, no hay argumentación lógico-deductiva sino valorativa o axiológica. Un principio, por razón de su mayor importancia desplaza a otro; pero esto no impedirá que en otro caso pueda aplicarse el principio desplazado o que el resultado de valorar la concurrencia entre los mismos principios sea diferente. Es en esta función donde los principios y reglas manifiestan una dimensión axiológica que, en la práctica, orienta tanto el conocimiento como la interpretación y la aplicación de las normas del sistema jurídico. 


\section{REFERENCIAS BIBLIOGRÁFICAS}

1.- ALEXY., R.,

Teoría de los derechos fundamentales, trad. De E. Garzón, Centro de Estudios Constitucionales, Madrid, 1993.

2.- ALEXY, R.,

"Sistema jurídico, principios jurídicos y razón práctica", trad. de Manuel Atienza, Doxa núm. 5, Alicante, 1989.

3.- ATIENZA, M., RUIZ MANERO, J.,

Las piezas del Derecho. Teoría de los enunciados jurídicos. Editorial, Isegoría 1999

4.- CANARIS, CLAUS-WILHELM,

Derechos Fundamentales y Derecho Privado, trad. Nueva York 1999

5.- CARRIO, GENARO.R.,

Principios jurídicos y positivismo jurídico, Abeledo Perrot, Buenos Aires, 1970.

6.- DWORKIN RONALD,...

La Filosofía del Derecho trad. de J. Sáinz de los Terreros, Fondo de Cultura Económica, México D.F., 1980.

7.- DWORKIN, RONALD.,

Los derechos en serio. Editorial Ariel 2012

\section{8.- GARCIA FIGUEROA ALFONSO.}

Principios y positivismo jurídico. Editorial, Centro de Estudios Constitucionales, 1998
9.- GUASTINI, RICARDO.,

Distinguiendo: Estudios de teoría y metateoría del Derecho. Editorial, Gedisa. 2009

10.- GUASTINI, R.,

"Principios de derecho y discrecionalidad judicial", Editorial, Digesto delle discipline civilistiche, Turín. 1996, vol. XIV

11.- HABERMAS, J.

"Justicia y legislación" en Facticidad y validez, sobre el Estado democrático de derecho en términos de teoría del discurso, trad. De M. Jiménez redondo, Trotta, Madrid, 1998.

12.- HAGE JAAP C.,

Reasoning with Rules. An Essay on Legal Reasoning and Its Underlying Logic (Razonamiento con reglas: un ensayo sobre el razonamiento jurídico y su lógica subyacente). Kluwer Academic Publishers, Dordrecht-Boston-London. 1997

13.- PEREZ LUÑO, A.E..,

Derechos humanos, Estado de Derecho y Constitución, Tecnos, Madrid.

14.- PRIETO SANCHIS LUIS.,

Sobre principios y normas. Problemas del razonamiento jurídico. Editorial, Centro de Estudios Constitucionales, Madrid 1992 
15.- PRIETO SANCHIS LUIS.,

Ley, principios, derechos, Dykinson, Madrid, 2002.

16.- RODRIGUEZ TOUBES JOAQUIN.

Principios, fines y derechos fundamentales. Dykinson. Madrid, 2000.
17.- RUIZ MANERO, J.,

“Principios jurídicos", en VV.AA., $\boldsymbol{E l}$ derecho y la justicia (E. Garzón, F.J. Laporta, eds.) Trotta, Madrid, 1996.

18.- RUIZ MANERO, J.,

"Principios Jurídicos". Trotta Madrid 1996 
